\title{
South African congenital disorders data, 2006 - 2014
}

\author{
V Lebese, BSc Hons; C Aldous, PhD; H L Malherbe, MSc \\ School of Clinical Medicine, College of Health Sciences, Nelson R Mandela School of Medicine, University of KwaZulu-Natal, Durban, \\ South Africa
}

Corresponding author: H L Malherbe (helen@hmconsult.co.za)

Background. The National Department of Health in South Africa (SA) routinely collects congenital disorder (CD) data for its national CD surveillance system. The current system has been implemented since 2006, but no reports on the data collected, methodology, achievements or challenges have been published to date.

Objectives. To ascertain the effectiveness of the current national CD surveillance system and its implementation.

Method. A descriptive, retrospective study using an audit of the current database was undertaken to evaluate the number of notifications received, types of CDs reported and the quality of reporting across SA for data received from 2006 to 2014.

Results. A total of 14571 notifications were received, including 13252 CDs and 1319 zero notifications, across all nine provinces. Commonly reported CDs included Down syndrome, cleft lip and palate, talipes equinovarus, neural tube defects and albinism.

Conclusions. The major challenges identified included erratic compliance by health facilities and a lack of healthcare providers trained in human genetics related to CDs. This has led to misdiagnosed and undiagnosed CDs, collectively resulting in under-reporting of cases by $>98 \%$ during the review period. Owing to limited human and financial resources, it is recommended that the surveillance system be modified into an electronic format. This should be piloted alongside relevant training in specific sentinel sites, to improve data coverage and quality for further evaluation.

S Afr Med J 2016;106(10):992-995. DOI:10.7196/SAMJ.2016.v106i10.11314

Congenital disorders (CDs) or birth defects are defined as abnormalities of structure or function, including metabolism, that are present from birth. ${ }^{[1]}$ Some are clinically obvious at birth, while others manifest later in life. CDs may be caused by genetic or partially genetic factors (preconception), non-genetic causes (postconception), a combination of these or unknown factors.

Surveillance of CDs is essential to providing reliable information for decision-making and policy development relevant to their care and prevention. ${ }^{[2]}$ Accurate data are required to demonstrate the true contribution of CDs to the burden of disease, which is imperative for any country.

CDs are the leading causes of infant and under- 5 child mortality in high-income countries, accounting for up to $28 \%$ of deaths. ${ }^{[3]}$ Surveillance systems in these countries, such as the National Birth Defects Prevention Network in the USA and the British Isles' Network of Congenital Anomalies, collate relevant data to determine the contribution of CDs to infant and child morbidity and mortality rates. ${ }^{[4,5]}$ Relevant health policy is developed in response to these observed data.

Insufficient epidemiological data hamper the provision of quality health services for people with CDs. This is a common challenge among low- and middle-income countries (LMICs) including South Africa (SA), necessitating the use of estimates through modelling. With the birth prevalence of CDs in LMICs greater than or equal to that of high-income countries, this highlights the importance of quality epidemiological data from comprehensive surveillance, and a functioning health system. ${ }^{[6,7]}$

CD surveillance was initiated in SA in 1980 through research on the teratogenic effects of water supplies undergoing new purification processes. Work by the Water Research Commission through the University of Cape Town ${ }^{[8]}$ provided the National Department of Health $(\mathrm{NDoH})$ with the basis of a national $\mathrm{CD}$ surveillance system. This system aimed to provide information on the extent of disabling conditions, and to plan prevention and rehabilitation programmes. ${ }^{[8]}$
Following the publication of the Policy Guidelines for the Management and Prevention of Genetic Disorders, Birth Defects and Disabilities ${ }^{[9]}$ in 2001, the national CD surveillance system was modified further by the NDoH with the introduction of the Birth Defect Notification Tool (BDNT) form. This article reports on the data emanating from the surveillance system implemented by the $\mathrm{NDoH}$ from 2006 to 2014.

\section{Methods}

Surveillance systems require clear goals and objectives to produce data that can be used to improve the general health of the public. The goals of the NDoH system were: $(i)$ to find out the incidence and prevalence rates of CDs; (ii) to determine priorities for intervention; (iii) to inform effective planning; (iv) to set objectives for prevention and care; and $(v)$ data evaluation and provision of feedback to provinces, health areas and health districts. ${ }^{[9]}$ The specific methodology used is outlined under subsequent headings. ${ }^{[10,11]}$

\section{Case definitions}

The case definitions define the parameters for the surveillance system. Various definitions were considered, including maximum age of patient and types of CDs (minor, major or only specific CDs). ${ }^{[4,10,11]}$ In 2001, the $\mathrm{NDoH}$ compiled a list of CDs (those that are identifiable or measurable within 24 hours of birth) to be monitored. These included neural tube defects (NTDs), Down syndrome, albinism, microcephaly, isolated cleft lip and/or palate and isolated hydrocephalus. ${ }^{[9]}$ In 2005, the National Guidelines for the Care and Prevention of the Most Common Genetic Disorders, Birth Defects and Disabilities were published. ${ }^{[12]}$ These outlined priority CDs that are common in SA and added talipes equinovarus, congenital infections and genetic deafness, blindness, physical handicap and mental retardation to the initial list of conditions for inclusion. 


\section{Case ascertainment methods}

Between 2006 and 2014, a total of 729 healthcare providers (HCPs) from all levels of care, including primary healthcare (PHC) centres, were trained in the use of the BDNT (D Tshikedi, personal communication, 2015). This training was undertaken through the Medical Genetics Education Programme (MGEP) for labour ward and outpatient ward staff to maximise opportunities for identifying CDs throughout the continuum of care. Completed BDNTs were submitted to the $\mathrm{NDoH}$ via the district and provincial departments of health (DoHs) on a monthly basis. Provincial co-ordinators were responsible for the collation and analysis of provincial data prior to submitting to the $\mathrm{NDoH}$. The identification and notification of the $\mathrm{CD}$ would preferably occur immediately following delivery, but, if undiagnosed within 24 hours of birth, notification could occur at any age, when the $\mathrm{CD}$ is identified. When no $\mathrm{CD}$ was identified at a specific facility within a reporting month, a zero notification tool was completed and submitted for the specific month using the same method. The BDNT was completed by various HCPs including registered nurses, medical doctors, genetic counsellors or clinical geneticists, if available. Only identified CDs were reported (preliminary diagnoses) while laboratory or other investigations were undertaken for confirmation.

\section{Pregnancy outcomes}

CDs on all pregnancy outcomes were to be monitored, including live births, stillbirths, terminations of pregnancy (TOP) and miscarriages.

\section{Classification/coding}

Although most countries opt to use the International Classification of Diseases, 10th revision (ICD-10), the $\mathrm{NDoH}$ developed a tailored coding system, capturing each $\mathrm{CD}$ using Microsoft Access and exporting the data to Microsoft Excel (USA). Each CD case was assigned a unique identifying number in the sequence received in that year, e.g. the first reported CD for 2006 was coded as 001/06. The system was implemented from May 2006

\section{Results}

Implementation of the BDNT system by the health facilities was slow and erratic. The total number of notifications received from 2006 to 2014 was 14 571, of which 13252 were CDs and 1319 were zero notifications.

National reporting (Table 1) peaked in 2011 with a total of 2401 cases reported, which was $2.92 \%$ of the expected number of $\mathrm{CD}$ cases based on modelled figures. ${ }^{[13,14]} \mathrm{In}$ 2014, the lowest number of CDs to date (612)

Table 1. CDs reported in SA for 2006 - 2014 compared with expected numbers based on modelled estimates

\begin{tabular}{lllll}
\hline Year & $\begin{array}{l}\text { Zero } \\
\text { notifications, } \boldsymbol{n}\end{array}$ & $\begin{array}{l}\text { CD } \\
\text { notifications, } \boldsymbol{n}\end{array}$ & $\begin{array}{l}\text { Expected CD } \\
\text { notifications, }{ }^{*} \boldsymbol{n}\end{array}$ & $\begin{array}{l}\text { Actual notifications as } \\
\text { \% of expected }\end{array}$ \\
\hline 2006 & 77 & 647 & 78201 & 0.83 \\
2007 & 109 & 1338 & 79020 & 1.69 \\
2008 & 112 & 1449 & 79914 & 1.81 \\
2009 & 135 & 1854 & 80829 & 2.29 \\
2010 & 89 & 1745 & 81680 & 2.14 \\
2011 & 387 & 2401 & 82349 & 2.92 \\
2012 & 232 & 2174 & 83118 & 2.62 \\
2013 & 119 & 1032 & 83821 & 1.23 \\
2014 & 59 & 612 & 84461 & 0.72 \\
Total & 1319 & 13252 & 733393 & 1.81 \\
${ }^{*}$ Based on modelled/estimated figure of $6.8 \%$ of live births affected by CD per annual number of births. ${ }^{[3,341}$
\end{tabular}

Table 2. CDs notified per province, 2006 - 2014, $n$

\begin{tabular}{lllllllllll}
\hline Province & $\mathbf{2 0 0 6}$ & $\mathbf{2 0 0 7}$ & $\mathbf{2 0 0 8}$ & $\mathbf{2 0 0 9}$ & $\mathbf{2 0 1 0}$ & $\mathbf{2 0 1 1}$ & $\mathbf{2 0 1 2}$ & $\mathbf{2 0 1 3}$ & $\mathbf{2 0 1 4}$ & Total \\
\hline Eastern Cape & 35 & 39 & 72 & 24 & 98 & 194 & 174 & 64 & 28 & 728 \\
Free State & 41 & 45 & 27 & 63 & 99 & 159 & 191 & 90 & 29 & 744 \\
Gauteng & 91 & 162 & 104 & 13 & 244 & 404 & 396 & 165 & 21 & 1600 \\
KZN & 231 & 732 & 790 & 1396 & 1116 & 1287 & 930 & 408 & 329 & 7219 \\
Limpopo & 32 & 105 & 72 & 81 & 25 & 88 & 106 & 11 & 21 & 541 \\
Mpumalanga & 99 & 72 & 27 & 93 & 34 & 72 & 148 & 203 & 89 & 837 \\
North West & 78 & 81 & 113 & 53 & 41 & 110 & 59 & 3 & 0 & 538 \\
Northern Cape & 40 & 65 & 110 & 62 & 61 & 50 & 150 & 81 & 95 & 714 \\
Western Cape & 0 & 37 & 134 & 69 & 27 & 37 & 20 & 7 & 0 & 331 \\
Total & 647 & 1338 & 1449 & 1854 & 1745 & 2401 & 2174 & 1032 & 612 & 13252
\end{tabular}

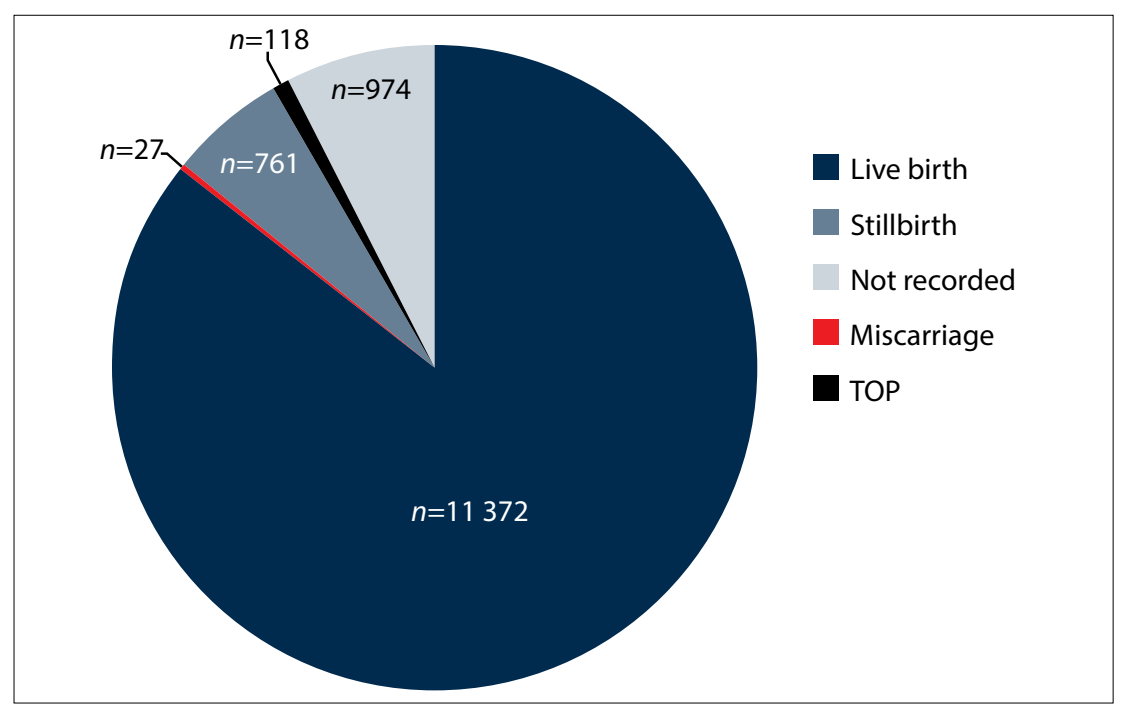

Fig. 1. Pregnancy outcomes of reported CDs as reported by facilities.

was reported, indicating under-reporting of more than $99 \%$.

Table 2 shows the distribution of $\mathrm{CD}$ notification tools received per province.
KwaZulu-Natal (KZN) recorded the highest number of reported CDs for every year of the review period and in total, with 7219 notifications (54.47\%). The Western 
Cape reported the least notifications in total $(n=331,2.50 \%)$.

Pregnancy outcomes as reported by health facilities are shown in Fig. 1. Early identification and diagnosis are vital to enable appropriate care and management of the affected infant should the mother choose to continue with the pregnancy. Of the 13252 reported CDs, $85.81 \%(n=11372)$ were live births, $5.74 \%(n=761)$ stillbirths, $0.89 \%(n=118)$ TOP and $0.20 \%(n=27)$ miscarriages. For $7.35 \%$ ( $n=974$ ) of notifications the pregnancy outcome was not indicated.

CDs may be identified prenatally or post delivery. Identifying CDs during pregnancy facilitates early identification of different approaches to management of the affected pregnancy, including choice of TOP. ${ }^{[9]}$ The majority $(85.81 \%)$ of infants affected by a $\mathrm{CD}$ in this study were born alive. However, the number of live births with a CD identified prenatally was only documented in $0.89 \%$ of cases, where patients opted for TOP following prenatal diagnosis.

During the review period, priority $\mathrm{CDs}^{[12]}$ contributed $35.92 \%(n=4760)$ of the total of 13252 CDs reported (Fig. 2). The leading CDs reported were Down syndrome $(n=1236,25.97 \%)$, talipes equinovarus $(n=1087,22.84 \%)$, cleft lip and/or palate $(n=943,19.81 \%)$ and NTDs ( $n=787,16.53 \%)$ (Table 3). Fetal alcohol syndrome (FAS) was the least reported, with 73 (1.54\%) cases.

Other reported cases that were not common priority CDs were categorised as 'other congenital disorders' (OCDs). These totalled $8492(64.08 \%)$, of which only 1497 (17.63\%) were diagnosed. Where OCDs could not be diagnosed but clear abnormalities were observed, the abnormal organ/system was reported with a description. Abnormalities of the hands (mainly polydactyly) were the most commonly reported ( $n=1775,20.90 \%)$, followed by those of the skull ( $n=659,7.76 \%)$ and the heart $(n=574,6.76 \%)$. Least reported were abnormalities of the skin with only nine cases $(0.11 \%)$ that were only monitored from 2013. Abnormalities may be isolated or occur on multiple organs or structures. Multiple systems/structure CDs accounted for 1360 $(16.02 \%)$ of the reported OCDs.

\section{Discussion}

The success of any congenital defects notification system or surveillance system depends upon three factors: ( $i$ ) training of HCPs and their ability to apply the acquired knowledge; (ii) the presence of a local (provincial) co-ordinator; and (iii) the compliance of HCPs with the surveillance system. The provincial co-ordinators are pivotal in this process owing to their dual training and

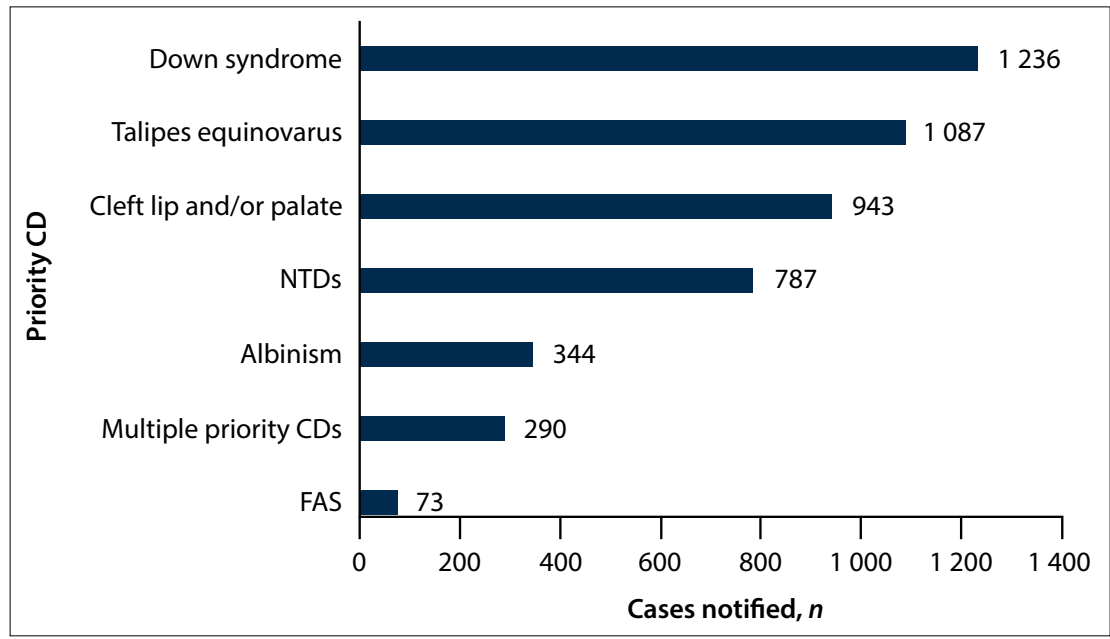

Fig. 2. Number of priority CDs notified, 2006 - 2012.

data collection and/or surveillance responsibilities. ${ }^{[9]}$

All nine provinces in SA should be implementing the BDNT, but current compliance is erratic and inadequate. The $13252 \mathrm{CD}$ notifications reported for the period 2006 2014 are only $1.81 \%$ of the expected total of 733393 , based on modelled estimates (Table 1). ${ }^{[13,14]}$ This implies under-reporting by $98.19 \%$ during this 8 -year period. In the last 2 years of the review period, reporting has dropped notably since implementation began in 2006, with the lowest figures reported in 2014.

KZN is the province with the second largest population in the country and reported an average of $54.47 \%$ of the total CD notification. ${ }^{[13]}$ KZN's initial success can be attributed to the presence of an active provincial co-ordinator and consistent training of HCPs via MGEP. The national decline in data from 2012 may be attributed to the loss of a highly effective KZN provincial co-ordinator who remains unreplaced to date. This has impacted negatively both on the management of the provincial genetic services and on data flow for 2012 and subsequent years.

In the Western Cape, where the fewest CDs were reported, provincial co-ordinators are also responsible for other maternal and child health programmes. Although genetic services are available at different facilities, co-ordination is lacking. This negatively impacts on the co-ordination of HCP training and data flow from facilities to the $\mathrm{NDoH}$ via the province, evidenced by only 331 CDs reported in total for 2006 - 2014.

In Gauteng, the province with the largest population between 2002 and 2015, the number of CDs notified peaked in 2011 $(n=404)$. However, with 200443 births recorded in Gauteng in 2011, ${ }^{[15]}$ modelled
Table 3. Other CDs notified, 2006 - 2014

\begin{tabular}{ll}
\hline Other CDs & $\boldsymbol{n}(\%)$ \\
\hline Named diagnosis & $1497(17.63)$ \\
Abdomen & $461(5.43)$ \\
Arms & $88(1.04)$ \\
Chest & $81(0.95)$ \\
Face & $464(5.46)$ \\
Feet & $156(1.84)$ \\
Gastrointestinal tract & $498(5.86)$ \\
Genitals & $379(4.46)$ \\
Hands & $1775(20.90)$ \\
Heart & $574(6.76)$ \\
Legs & $391(4.60)$ \\
Skin & $9(0.11)^{*}$ \\
Skull & $659(7.76)$ \\
Urinary system & $100(1.18)$ \\
Multiple systems/structure & $1360(16.02)$ \\
Total OCDs & $8492(100)$ \\
${ }^{*}$ Monitored from 2013. & \\
&
\end{tabular}

data indicate a minimum of 13630 births (6.8\%) with a CD in the province, with 3571 of those identifiable within the first 24 hours of life. ${ }^{[14]}$ This indicates significant underreporting (97.04\%) in Gauteng in 2011.

Of the total of 13252 CDs reported nationally during the 8-year period of this study, only $35.92 \%$ (4 760) were common priority conditions. NTDs contributed the greatest single group of CDs (16.53\%), including anencephaly, encephalocele and spina bifida. More than half $(52.78 \%)$ of the reported CDs were undiagnosed. This highlights the importance of training HCPs in identifying, diagnosing and referring patients for appropriate $\mathrm{CD}$ management and care. 
FAS was the least-reported CD, with only 73 reported cases (1.53\%). This is unsurprising as the diagnosis of FAS is extremely challenging, especially in the neonatal period, and requires clinical examination of features by a trained HCP. ${ }^{[16]}$ Of the 73 FAS cases reported, 44 were diagnosed at birth and 29 were diagnosed later in the continuum of care. These data do not align with literature reporting FAS prevalence in SA as the highest documented globally, suggesting that FAS is grossly underreported via the BDNT.

\section{Challenges}

The provincial DoHs are guided by the $\mathrm{NDoH}$ but function autonomously. Nationally developed policies are implemented by the provinces in a top-down approach. The 2001 Policy Guidelines were developed in collaboration with the provinces and relevant stakeholders, but implementation of this policy, including the BDNT, has been problematic. Despite all provinces implementing the tool, erratic compliance by the districts is a challenge for data collection. Without a zero notification from a facility for a particular month, it is not known whether this is due to non-submission of forms or because no CDs were detected during that period.

By the end of 2012, although MGEP training had been conducted in every district in SA, only 46 of the 52 districts were submitting BDNTs. While some facilities lack a human genetics-trained HCP, there is also a lack of continued training for additional HCPs throughout the continuum of care with no relevant follow-up support provided. As a result, many CDs continue to be misdiagnosed or remain undiagnosed. This prevents a notification from being submitted and, more significantly, precludes the patient from receiving the appropriate care and treatment.

A further constraint is that only the public health sector is involved in national surveillance, with no notifications received from private healthcare. This further exacerbates the underreporting of CDs. The data collection process is also affected by the poor quality of the data received, including the submission of incomplete forms, illegibile handwriting and late submission. The greatest challenge is that the $\mathrm{NDoH}$ data capture system is not compatible with any other surveillance system, including the ICD-10 - preventing data comparison with other programmes. ${ }^{[9]}$

A well-functioning CD surveillance system could prove particularly useful when new pathogens appear. The Zika virus (ZIKV) has recently been implicated as a cause for microcephaly in newborns. The ZIKV vector is present in SA along the eastern seaboard, breeding in Strelitzia leaves, and there is anecdotal evidence that microcephaly has increased in incidence over the years. However, without accurate CD surveillance data, a possible link with ZIKV cannot be shown. ZIKV may have had a similar impact on CDs along the eastern reaches of SA as in Brazil, because of shipping routes east of Africa. ${ }^{\left[{ }^{17]}\right.} \mathrm{A}$ comprehensive, functioning surveillance system would have identified any change in trend of microcephaly incidence and contributed to further research in this area.

\section{Recommendations}

Strengthening surveillance of CDs in SA requires the consideration of new avenues. The approach taken to date in SA is the use of a population-based surveillance programme, in which data are collected from an entire source population within a defined time period. ${ }^{[11]}$ This approach is not working, because of limited resources (human and financial) and the current state of the medical genetic services in the country. ${ }^{[14]}$ For the future, it is recommended that a hospital-based surveillance programme be implemented, similar to the Perinatal Problem Identification Program (PPIP) model, where data are collected from specific hospitals and facilities in a defined geographical area. ${ }^{[10,11]}$ A revised BDNT should be developed using an electronic application (app)-based approach to collect data, eliminating the current paper-based method.

To improve the quality and collection of data, capacity building of HCPs in PHC settings should be reintroduced and strengthened. Health facilities responsible for the management and treatment of patients with CDs require improved support to enable them to notify CDs. Healthcare providers at these facilities are at the core of the system, reporting on confirmed diagnoses following further investigations.

Improved integration is required, and the $\mathrm{NDoH}$, provinces, facilities and other roleplayers need to work together to improve CD data collection countrywide. The private healthcare sector should also be incorporated into the notification system. Laboratory services and their contribution to CD surveillance need improving and non-governmental organisations should be permitted to play a greater role in the collection of evidence-based data through patient registries, to more accurately reflect the true disease burden of CDs in SA.

\section{Conclusions}

The current national surveillance of CDs implemented through the BDNT is resulting in poor quality and quantity of data. This needs to be rectified to ensure a higher quality of $\mathrm{CD}$ observational data, to highlight the growing contribution of CDs to the disease burden in the country. Greater commitment is required to comprehensively collect and analyse CD data and apply these findings in policy development and implementation, by improving medical genetic services for those affected or at risk of CDs. Such improved surveillance would be an important step in response to World Health Assembly Resolution 63.17 of $2010 .^{[2]}$

Acknowledgement. We thank the $\mathrm{NDoH}$ for their assistance in fasttracking the data for this research.

\footnotetext{
1. World Health Organization, March of Dimes. Management of birth defects and haemoglobin disorders. Report of a joint WHO-March of Dimes meeting, Geneva, Switzerland. Geneva: WHO, 2006 http:/ www.who.int/genomics/publications/WHO-MODreport-final.pdf (accessed 10 May 2014).

World Health Assembly. Resolution 63.17. Birth Defects. Geneva: WHA, 2010. http://apps.who.int/gb/ ebwha/pdf_files/WHA63/A63_R17-en.pdf (accessed 2 September 2014).

3. World Health Organization. World Health Statistics 2015. Geneva: WHO, 2015. http://www.who.int/ gho/publications/world_health_statistics/2015/en/(accessed 24 February 2016).

gho/publications/world_health_statistics/2015/en/(accessed 24 February 2016).
4. Sever LE, ed. Guidelines for Conducting Birth Defects Surveillance. Atlanta, USA: National Birth Defect Prevention Network, 2004.

5. Kurinczuk JJ, Hollowell J, Boyd PA, Oakley L, Brocklehurst P, Gray R. The contribution of congenital anomalies to infant mortality. Inequalities in Infant Mortality Project Briefing Paper 4. Oxford: National Perinatal Epidemiology Unit, University of Oxford, 2010.

6. Bale JR, Stoll BJ, Lucas AO, eds. Committee on Improving Birth Outcomes. Reducing Birth Defects: Meeting the Challenge in the Developing World. Washington, DC: National Academies Press, 2003. Christianson A, Modell B. Medical genetics in developing countries. Annu Rev Genomics Hum Genet 2004;5:219-265. DOI:10.1146/annurev.genom.5.061903.175935

8. Sayed AR, Nixon J, Bourne D. Birth Defects Surveillance System. Final Technical Report. Prepared for the Department of Health by School of Public Health and Family Medicine, University of Cape Town, South Africa, 2005

9. National Department of Health. Policy Guidelines for the Management and Prevention of Genetic Disorders, Birth Defects and Disabilities. Pretoria: NDoH, 2001

0. Luquetti DV, Koifman RJ. Surveillance of birth defects: Brazil and the US. Cien Saude Colet . Luquetti DV, Koifman RJ. Surveillance of birth defects: Brazil and
2011;16(Suppl 1):777-785. DOI:10.1590/S1413-81232011000700008

1. World Health Organization, Centers for Disease Control, International Clearing House for Birth World Health Organization, Centers for Disease Control, International Clearing House for Birth
Defects Surveillance and Research. Birth Defects Surveillance: A Manual for Programme Managers. Geneva: WHO, 2014

12. National Department of Health. National Guidelines for the Care and Prevention of the Most Common Genetic Disorders, Birth Defects and Disabilities. Pretoria: NDoH, 2005.

3. Statistics South Africa. Mid-year Population Estimates: 2015. Statistical Release P0302. Pretoria: SSA, 2015. http://www.statssa.gov.za/publications/P0302/P03022015.pdf (accessed 18 February 2016).

14. Malherbe $\mathrm{H}$, Christianson AL, Aldous C. Need for services for the care and prevention of congenital disorders in South Africa as the country's epidemiological transition evolves. S Afr Med J 2015;105(3):186-188. DOI:10.7196/SAMJ.9136

15. Statistics South Africa. Recorded Live Births 2014. Statistical Release P0305. Pretoria: SSA, 2015. http://www.statssa.gov.za/publications/P0305/P03052014.pdf (accessed 16 August 2016).

16. Bertrand J Floyd RL, Weber MK, et al. National Task Force on FAS/FAE. Fetal Alcohol Syndrome: Guidelines for Referal and Diagnas., Nato 2004.

17. Jansen van Vuren P, Weyer J, Kemp A, Dermaux-Msimang V. Is South Africa at risk for Zika virus disease? S Afr Med J 2016;106(3):232-233. DOI:10.7196/SAMI.2016.v106i3.10615
} 